\title{
CtBP2 is an independent prognostic marker that promotes GLI1 induced epithelial-mesenchymal transition in hepatocellular carcinoma
}

\author{
Xin Zheng ${ }^{1, *}$, Tao Song ${ }^{1, *}$, Changwei Dou ${ }^{1}$, Yuli Jia ${ }^{1}$, Qingguang Liu ${ }^{1}$ \\ ${ }^{1}$ Department of Hepatobiliary Surgery, the First Affiliated Hospital of Xi'an Jiaotong University, Xi'an, Shaanxi 710061, China \\ "These authors have contributed equally to this work \\ Correspondence to: \\ Qingguang Liu, e-mail: raymondzhengxin@foxmail.com \\ Keywords: C-terminal binding protein 2, C†BP2, GLI family zinc finger 1, GLI1, Snail Family Zinc Finger 1, epithelial-mesenchymal \\ transition, EMT, Hepatocellular carcinoma, HCC \\ Received: November 12, $2014 \quad$ Accepted: December 15, $2014 \quad$ Published: February 19, 2015
}

\section{ABSTRACT}

C-terminal binding protein 2 (CtBP2) is a transcriptional co-repressor that promotes cancer cell migration and invasion by inhibiting multiple tumor suppressor genes that contribute to cell mobility and adhesion. In this investigation, we showed thatCtBP2 expression was increased significantly in HCC tissues when compared to matched normal adjacent liver tissues. We also showed that CtBP2 expression is associated with worse HCC patient prognosis after liver resection. CtBP2 overexpression induced epithelial-mesenchymal transition (EMT) in Huh7 cells and, correspondingly, silencing CtBP2 suppressed EMT in MHCC97H cells. ChIP assays revealed that GLI1 increased CtBP2 transcription by directly binding its promoter. Furthermore, interaction of CtBP2 and Snail Family Zinc Finger 1 (SNAI1), both of which were found to be positively regulated by GLI1, was confirmed by Co-IP assay. SNAI1 knockdown revealed that SNAI1 was essential for CtBP2 induction of the EMT phenotype of HCC cells, and CtBP2 knockdown reversed GLI1-SNAI1 driven EMT in Huh7 cells. Finally, in vivo experiments demonstrated that enhanced CtBP2expression promoted HCC xenograft growth and induced EMT. In conclusion, CtBP2 may serve as a prognostic marker for post liver resection HCC and may play a role during GLI1driven EMT as a transcriptional co-repressor of SNAI1.

\section{INTRODUCTION}

Hepatocellular carcinoma (HCC) is the most common primary liver cancer subtype [1] and one of the major causes of mortality in developing countries [2]. Current curative therapies, including liver resection and liver transplantation, have a high rate of post-surgical recurrence [3]. HCC recurrence occurs because of both primary tumor metastatic dissemination prior to surgery and new neoplasm development in the remaining liver tissues after surgery. Because of the high rate of recurrence, the postsurgical prognosis of $\mathrm{HCC}$ patients is extremely poor. Therefore, better understanding of the molecular mechanisms underlying HCC recurrence and metastasis and identification of biomarkers that can predict postsurgical HCC outcome is urgently needed.
C-terminal binding protein (CtBP) was first established as a phosphoprotein bound to the $\mathrm{C}$ terminal of the E1A protein [4]. The CtBP family consists of two genes, CtBP1 and CtBP2, which are conserved among both vertebrates and invertebrates [5]. CtBPs reportedly function primarily as transcriptional co-repressors and play important roles in development and tumorigenesis [6-9]. CtBPs have also been demonstrated to function as context-dependent transcriptional activators $[10,11]$. A growing body of evidence indicates that CtBP2 is aberrantly upregulated in a variety of cancers and that its expression promotes cancer progression. Zhang et al. reported that $\mathrm{CtBP} 2$ was overexpressed in prostate cancer tissues and associated with poor outcome and several indicators of malignancy, including elevated serum PSA levels, advanced tumor stages and higher Gleason 
scores. Zhang et al. also reported that CtBP2 expression promoted prostate cancer cell proliferation through c-Myc signaling [12]. Barroilhet et al. found that CtBP2 was aberrantly elevated in human ovarian tumors and that CtBP2 expression accelerated tumor cell growth and mobility [13]. Similar findings have also been reported in breast cancer studies that examined CtBP 2 expression $[14,15]$. However, the mechanism governing CtBP2 overexpression in cancer remains unclear.

Glioma-associated oncogene 1 (GLI1) is a downstream transcription factor in the hedgehog signaling pathway that reportedly accelerates the progression of various cancers, including basal cell carcinoma [16], colon cancer [17], medulloblastoma [18], gastric cancer [19] and pancreatic carcinoma [20]. We previously reported GLI1 overexpression in HCC tissues and were able to predict rapid tumor recurrence after surgery using GLI1 expression levels. GLI1 consists of a conserved $\mathrm{C} 2 \mathrm{H} 2$ zinc finger DNA-binding domain that specifically interacts with a GACCACCCA-like motif in the promoters of various target genes. GLI1 mediates the expression of a variety of important cancer progression genes, including SNAI1 [21], ABCG2 [22], CYLD, DNMT [23], RegIV [24], AKT [25] and Caveolin-1 [26]. We demonstrated that aberrantly upregulated GLI1 promoted HCC progression by regulating epithelial-mesenchymal transition (EMT) through the GLI1 transcriptional target Snail Family Zinc Finger 1 (SNAI1) [21]. We found seven potential GLI1 binding sites in the CtBP2 promoter, which lead us to hypothesize that GLI1 overexpression might contribute to the upregulation of CtBP2 in HCC.

We report that $\mathrm{CtBP} 2$ expression was upregulated in the HCC tissues examined in this study, and that elevated CtBP2 expression was associated with elevated GLI1 expression and poor overall postsurgical survival. We verified that CtBP2 was directly upregulated by GLI1 in vitro, and demonstrated that the upregulated CtBP2 bound the PQDLSLK motif of SNAI1 in the nucleus. Additionally, the oncogenic Role of CtBP2 was validated using a HCC xenograft model. Therefore, the findings of this study indicate that CtBP2 might facilitate GLI1-SNAI1 induced EMT in HCC. This is the first report describing the mechanism underlying $\mathrm{CtBP} 2$ overexpression, identifying the role played by $\mathrm{CtBP} 2$ in EMT in HCC and showing CtBP2 is an effective predictive biomarker of patient outcome after HCC surgery.

\section{RESULTS}

\section{CtBP2 was overexpressed in $\mathrm{HCC}$ and overexpression predicted poor post-surgical outcomes}

We examined tumor tissues and adjacent liver tissues from $100 \mathrm{HCC}$ patients for CtBP2 expression by immunohistochemistry. We found that $\mathrm{CtBP} 2$ staining was strongest in the tumor cell cytoplasm, while nuclear staining was observed in relatively few cells (Figure 1A). CtBP2 expression was elevated in tumor cells when compared to benign tissues in $60 \%$ of the HCC tumors examined. Mann-Whitney $U$ analysis demonstrated that CtBP2 expression was significantly higher in HCC tissues when compared to adjacent liver tissues $(p<0.001$, Figure 1B). Clinical association analysis using the Spearman rank test demonstrated that CtBP2 overexpression in HCC tissues was positively correlated with tumor size $(p=0.039)$, venous infiltration $(p=0.003)$, Edmondson-Steiner Classification $(p<0.001)$ and the Tumor Node Metastasis (TNM) stage $(p=0.018)$.

Post-surgical follow-up information was collected from 87 of the original 100 HCC patients and Kaplan-Meier survival curves were constructed. The median overall survival was 14.8 months for HCC patients with elevated tumor tissue CtBP2 expression (High CtBP2 Group), whereas the median overall survival was 58.3 months for HCC patients with lower CtBP2 levels in adjacent liver tissues (Low CtBP2 Group). The three-year survival rate was 30.9\% in the High CtBP2 Group compared to $70.7 \%$ in the Low CtBP2 Group. Similarly, patients in the High CtBP2 Group (23.6\%) had a reduced five-year survival rate when compared to patients from the Low CtBP2 Group (41.9\%). Overall survival curve comparisons indicated that the High CtBP2 Group patients had a significantly worse prognosis when compared to patients from the Low CtBP2 Group (HR $=3.071 ; 95 \%$ CI: $1.357,6.951 ; p=0.007$; Figure $1 \mathrm{C}$ ). Univariate analysis demonstrated that venous infiltration, higher EdmondsonSteiner classification, advanced TNM staging and higher $\mathrm{CtBP} 2$ expression in tumor tissues were the poor prognosis factors (Table 2). Moreover, multivariate Cox proportionalhazard regression analysis indicated that venous infiltration, advanced TNM staging and higher CtBP2 expression in tumor tissues were independent prognostic factors (Table 2). These data indicated that CtBP2 overexpression in $\mathrm{HCC}$ tissues was a predictor of poor survival outlook after liver resection. To investigate whether there was a correlation between CtBP2 and GLI1 protein expression in HCC tissues, we conducted immunohistochemical (IHC) analysis of GLI1 expression in the clinical samples. As shown in Figure 1D, GLI1 was upregulated in HCC tissues when compared to adjacent liver tissues, which was consistent with the results of previous investigations [21, 26]. Similarly, SNAI1 expression was significantly elevated in HCC tissues when compared to adjacent liver tissues (Figure 1E). Spearman rank analysis positively associated elevated GLI1 expression with CtBP2 and SNAI1 in HCC tissues ( $r=0.701, p<0.001$, Figure 1F). This result raised the possibility that GLI1 expression could be related to the overexpression of both $\mathrm{CtBP} 2$ and SNAI1 in $\mathrm{HCC}$.

\section{CtBP2 expression in $\mathrm{HCC}$ cell lines}

To examine CtBP2 expression in HCC cell lines and to identify the most appropriate $\mathrm{HCC}$ cell model for 
A

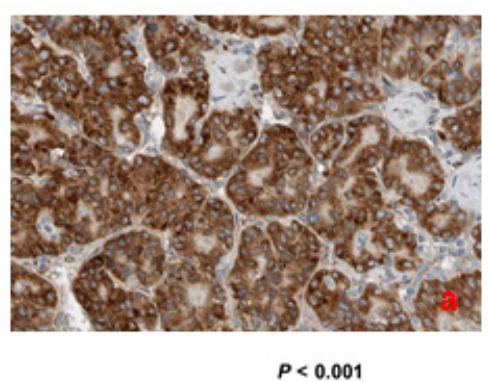

B

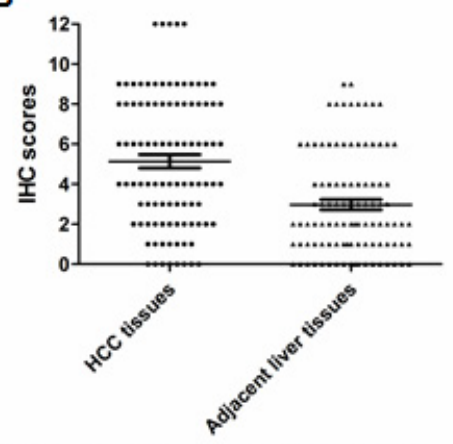

D

E
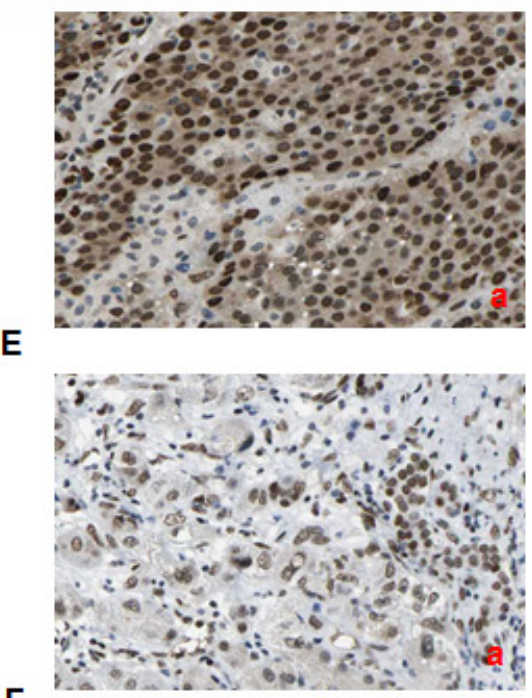

F

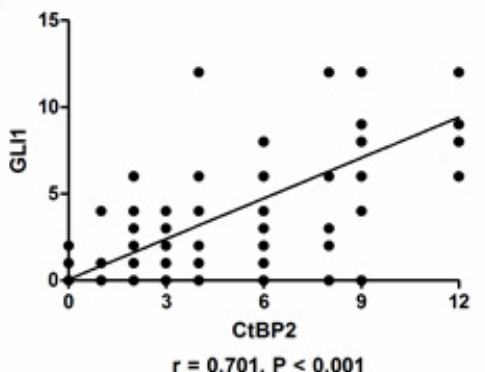

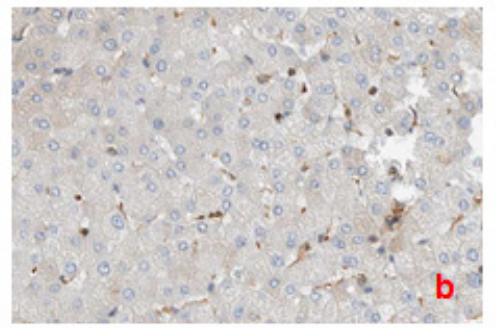

... Low CtBP2

- High CtBP2

C

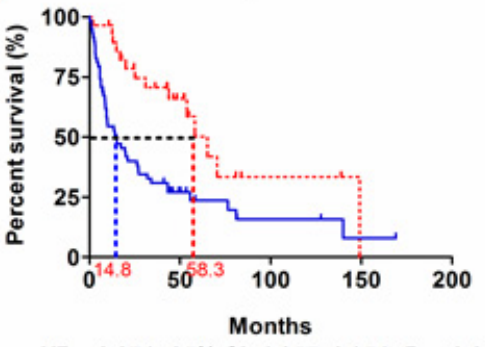

$\mathrm{HR}=3.071 ; 95 \% \mathrm{Cl}: 1.357,6.951 ; \mathrm{P}=0.007$
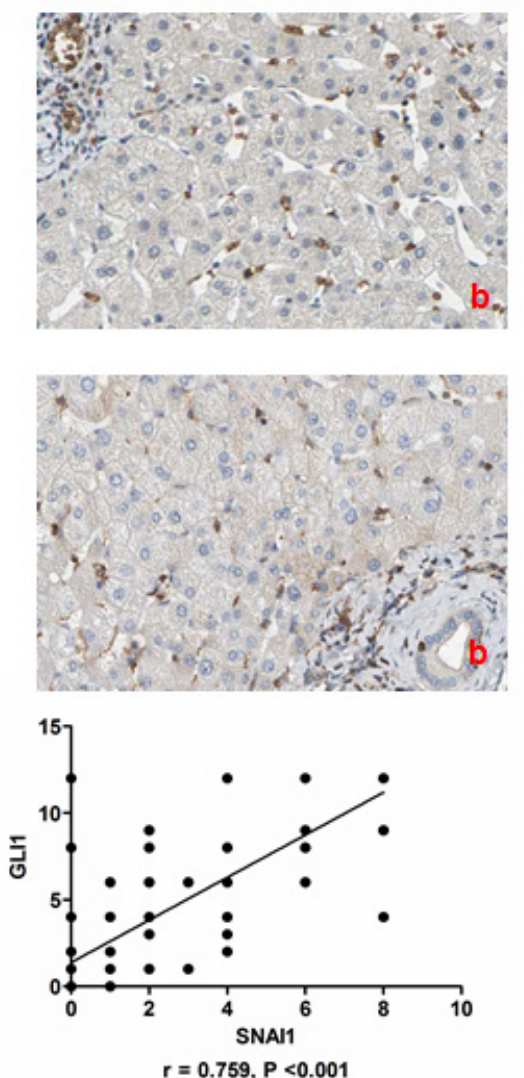

Figure 1: CtBP2 expression was upregulated in HCC tissues and associated with poor prognosis after liver resection. (A) CtBP2 was expressed mainly in the cytoplasm of tumor cells and CtBP2 expression was notably higher in HCC tissues (a) when compared to matched normal adjacent liver tissues (b). (B) The Mann-Whitney $U$ test confirmed that CtBP2 expression was significantly higher in HCC tissues than adjacent liver tissues from $100 \mathrm{HCC}$ patients $(p<0.001)$. (C) Kaplan Meier overall survival curves indicated that the High CtBP2 Group had an obviously shortened post-surgical survival time when compared to the Low CtBP2 Group (HR = 3.071; 95\% CI: 1.357, 6.951; $p=0.007$ ). (D) There was more GLI1 expression in HCC tissues (a) compared to matched normal adjacent liver tissues (b), and most of the GLI1-positive cells showed nuclear staining. (E) SNAI1 expression was notably increased in HCC tissues by IHC (a) when compared to matched adjacent healthy liver tissues (b). (F) The Spearman rank test indicated that GLI1 expression correlated positively with CtBP2 expression ( $r=0.701, p<0.001$, left panel) and SNAI1 $(r=0.759, p<0.001$, right panel $)$ in HCC tissues, respectively. 
Table 1: Correlation between clinicopathological characteristics and CtBP2 overexpression in the tumor tissues of $100 \mathrm{HCC}$ patients

\begin{tabular}{|c|c|c|c|c|c|}
\hline \multicolumn{2}{|c|}{ Clinicopathological features } & \multicolumn{2}{|c|}{ No. of patients } & \multirow[t]{2}{*}{$x^{2}$} & \multirow[t]{2}{*}{$\mathrm{p}$} \\
\hline & & Higher CtBP2 & Lower CtBP2 & & \\
\hline \multirow[t]{2}{*}{ Age (year) } & $<50$ & 20 & 12 & 0.12 & 0.726 \\
\hline & $\otimes 50$ & 40 & 28 & & \\
\hline \multirow[t]{2}{*}{ Gender } & Male & 48 & 30 & 0.35 & 0.554 \\
\hline & Female & 12 & 10 & & \\
\hline \multirow[t]{2}{*}{ HBV infection } & Present & 50 & 32 & 0.18 & 0.671 \\
\hline & Absent & 10 & 8 & & \\
\hline \multirow[t]{2}{*}{$\begin{array}{l}\text { Serum AFP level } \\
(\mathrm{ng} / \mathrm{mL})\end{array}$} & $\nabla 400$ & 46 & 27 & 1.02 & 0.312 \\
\hline & $<400$ & 14 & 13 & & \\
\hline \multirow[t]{2}{*}{ Tumor size $(\mathrm{cm})$} & $\otimes 5$ & 51 & 27 & 4.28 & 0.039 \\
\hline & $<5$ & 9 & 13 & & \\
\hline \multirow[t]{2}{*}{ Liver cirrhosis } & Present & 56 & 33 & 2.88 & 0.089 \\
\hline & Absent & 4 & 7 & & \\
\hline \multirow[t]{2}{*}{ Venous infiltration } & Present & 30 & 8 & 9.17 & 0.003 \\
\hline & Absent & 30 & 32 & & \\
\hline \multirow[t]{2}{*}{$\begin{array}{l}\text { Edmondson-Steiner } \\
\text { Classification }\end{array}$} & $\mathrm{I}+\mathrm{II}$ & 6 & 21 & 21.99 & $<0.001$ \\
\hline & $\mathrm{III}+\mathrm{IV}$ & 54 & 19 & & \\
\hline \multirow[t]{2}{*}{ TNM stage } & $\mathrm{I}+\mathrm{II}$ & 38 & 34 & 5.59 & 0.018 \\
\hline & III + IV & 22 & 6 & & \\
\hline
\end{tabular}

Table 2: Cox regression analysis of the correlation between clinicopathological features and the overall postsurgical survival rate of HCC patients

\begin{tabular}{l|c|c|c|c|}
\hline \multirow{2}{*}{$\begin{array}{l}\text { Clinicopathological } \\
\text { features }\end{array}$} & \multicolumn{2}{c}{ Unvariate Analysis } & \multicolumn{2}{c}{ Multivariate Analysis } \\
\cline { 2 - 5 } & RR (95\% CI) & $\mathrm{p}$ Value & $3.562(1.750-4.895)$ & $\mathrm{p}$ Value \\
\hline Venous infiltration & $4.320(2.043-6.875)$ & 0.018 & $1.854(0.899-2.152)$ & 0.025 \\
\hline $\begin{array}{l}\text { Higher Edmondson- } \\
\text { Steiner classification }\end{array}$ & $2.322(1.252-3.210)$ & 0.003 & $1.989(1.228-3.788)$ & 0.010 \\
\hline $\begin{array}{l}\text { Advanced TNM } \\
\text { staging }\end{array}$ & $3.232(2.012-4.365)$ & 0.008 & $3.025(1.752-6.982)$ & 0.030 \\
\hline $\begin{array}{l}\text { Higher CtBP2 in } \\
\text { tumor tissue }\end{array}$ & $3.689(1.922-5.025)$ & 0.021 & & 0.001 \\
\hline
\end{tabular}

further investigations, we conducted qRT-PCR assays and Western blots to detect $\mathrm{CtBP} 2$ mRNA and protein expression, respectively, in Hep3B, PLC/PRF/5, HepG2, MHCC97H and Huh7 cells. All five HCC cell lines expressed different $\mathrm{CtBP} 2$ levels (Figure 2A). When compared to the Huh7 and Hep3B cell lines, CtBP2
mRNA expression was higher in MHCC97H, PLC/PRF/5 and HepG2 cells. CtBP2 protein expression, as measured by Western blotting, mirrored the mRNA expression in all five HCC cell lines (Figure 2B). The Huh7 and MHCC97H cell lines had the lowest and the highest CtBP2 expression levels, respectively. Therefore, we selected the Huh7 cell 


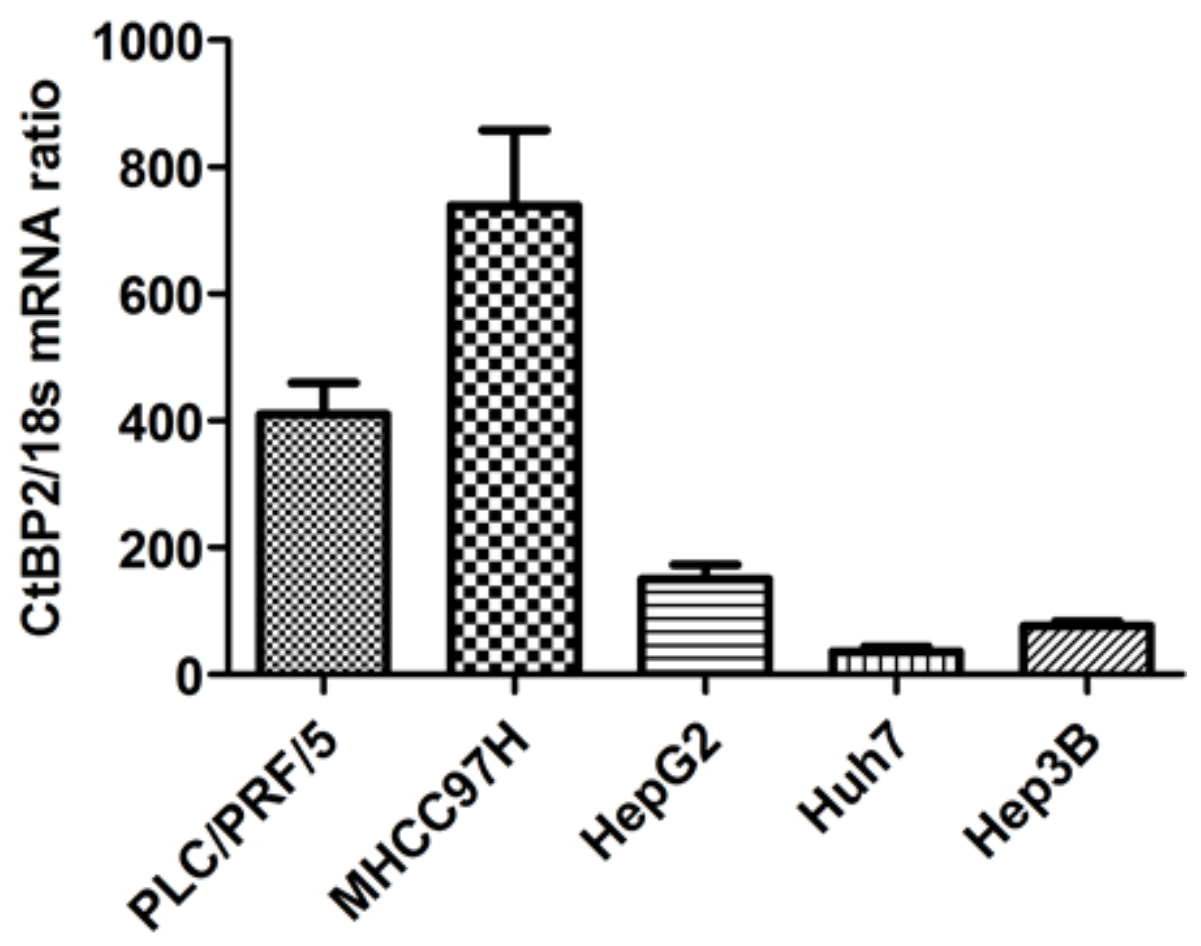

B
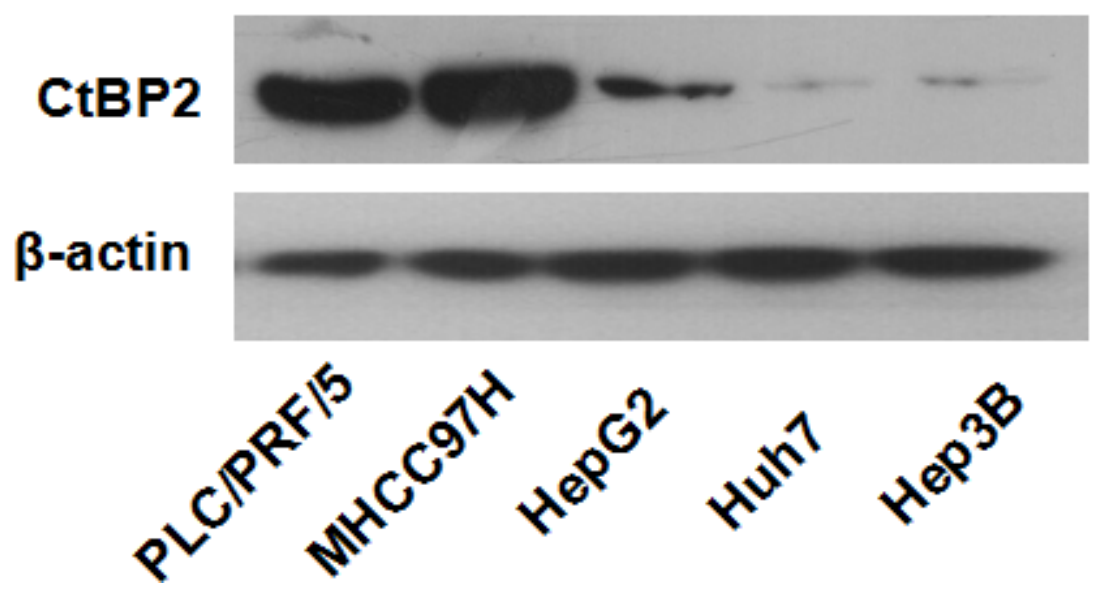

Figure 2: The expression of CtBP2 in HCC cell lines. (A) CtBP2 mRNA levels in five different HCC cell lines was assessed by qRT-PCR. (B) CtBP2 protein expression in five different HCC cell lines was examined by Western blotting.

line for the CtBP2 overexpression experiments in this study, and the MHCC97H cell line was used for the CtBP2 knockdown experiments.

\section{CtBP2 promoted HCC cell migration and invasion by inducing EMT}

To investigate the mechanism underlying CtBP2 promotion of $\mathrm{HCC}$ progression, we increased $\mathrm{CtBP} 2$ expression in Huh7 cells by stably transfecting the Huh7 cell line with a CtBP2 expressing plasmid. Successful transfection was confirmed by both qRT-PCR and Western blotting (Figure 3A). Wound healing assays demonstrated that the migration rate of Huh7 cells stably transfected with the CtBP2 expressing plasmid (Huh7 CtBP2 cells) was notably faster than Huh7 cells transfected with a vector plasmid control (Huh7 Vector cells) 24 and $48 \mathrm{~h}$ after scratching the cell layer (Figure 3B). To assess invasiveness, we conducted Transwell invasion assays and observed that Huh7 cell invasiveness was significantly increased by increased CtBP2 expression (Figure 3C). 
A
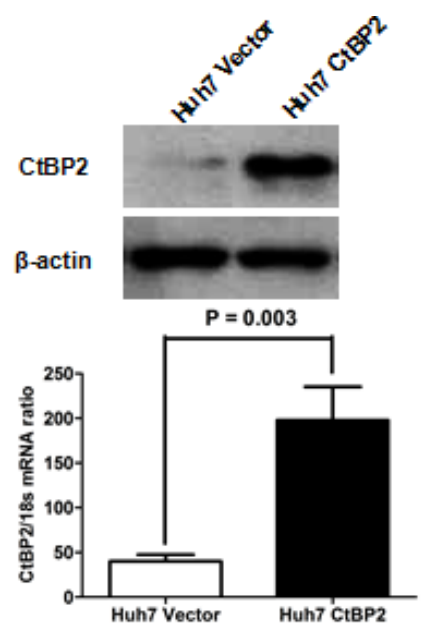

C
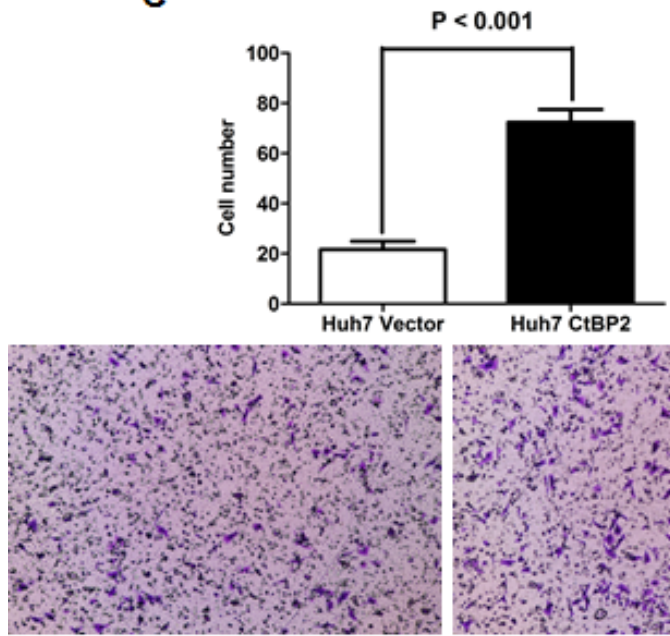

Huh7 Vector

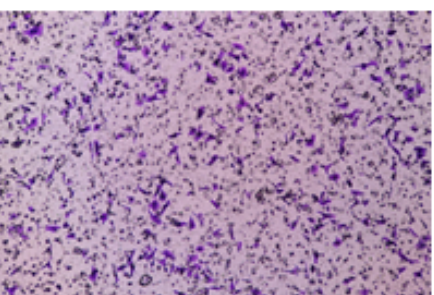

Huh7 CtBP2
B

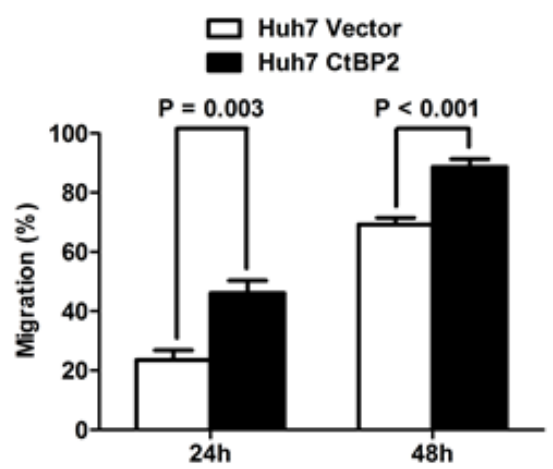

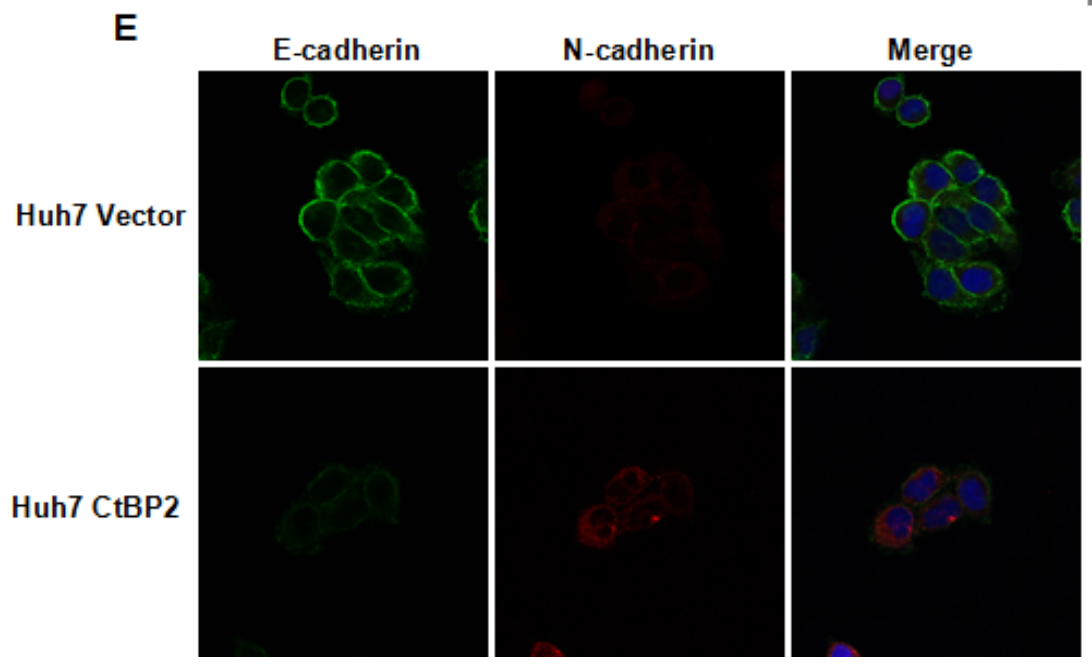

D

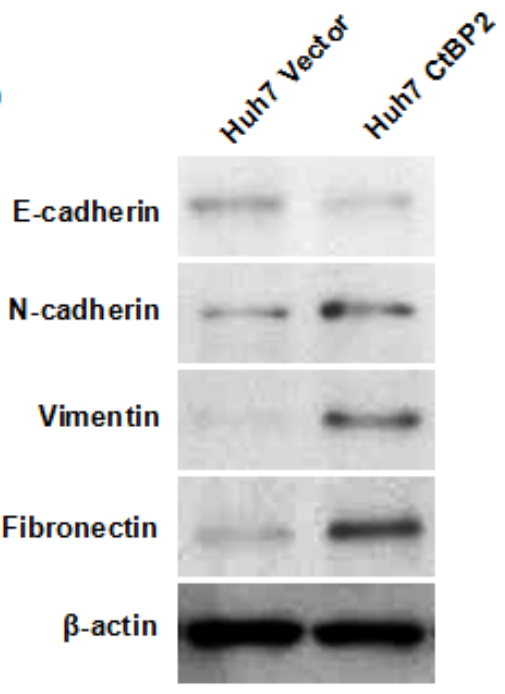

Figure 3: Elevated CtBP2 expression promoted Huh7 cell mobility and invasion by inducing EMT. (A) Both qRTPCR and Western blotting assays verified that CtBP2 expression was increased after stable transfection of CtBP2 expression plasmid. (B) The migration capacity of Huh7 cells, as assessed by wound healing assays, was enhanced by CtBP2 overexpression. (C) Transwell invasion assays demonstrated that elevating CtBP2 expression increased the invasive capability of Huh7 cells. (D) Overexpression of CtBP2 decreased E-cadherin expression and increased the expression of N-cadherin, Vimentin and Fibronectin in Huh7 cells, as measured by Western blotting. (E) Double immunofluorescence staining confirmed that CtBP2 overexpression resulted in the downregulation of E-cadherin and the upregulation of $\mathrm{N}$-cadherin in Huh7 cells. 
To investigate whether CtBP2 expression accelerated HCC cell mobility and invasiveness by inducing EMT, we examined the expression of EMT biomarkers in Huh7 cells using Western blotting and immunofluorescence staining. The biochemical markers examined included E-cadherin (which is expressed predominantly in epithelial cells), and N-cadherin, Vimentin and Fibronectin (which are expressed predominantly in mesenchymal cells). The Western blotting indicated that elevated CtBP2 expression attenuated E-cadherin expression, but increased N-cadherin, Vimentin and Fibronectin expression in Huh7 cells (Figure 3D). We performed double immunofluorescence staining to confirm the Western blotting experiments and observed reduced E-cadherin and elevated N-cadherin expression in Huh7 CtBP2 cells compared to the control Huh7 Vector cells (Figure 3E).

To verify the role of CtBP2 on EMT in HCC, we used $\mathrm{CtBP} 2$ targeted siRNAs to silence $\mathrm{CtBP} 2$ expression in $\mathrm{MHCC} 97 \mathrm{H}$ cells (Figure 4A). Consistent with the results in the Huh7 cell experiments, CtBP2 knockdown suppressed the migration and invasion of MHCC97H cells (Figure 4B and 4C). Additionally, we observed that CtBP2 suppression lead to E-cadherin upregulation in MHCC97 cells, but decreased the expression of N-cadherin, Vimentin and Fibronectin (Figure 4D). Taken together, these data strongly support the hypothesis that $\mathrm{CtBP} 2$ promotes $\mathrm{HCC}$ cell mobility and invasion by inducing EMT.

\section{GLI1 upregulated CtBP2 transcription by binding with its promoter}

Our preliminary investigations suggested that GLI1 plays a critical role in the EMT phenotype of HCC cells and the results of the current study strongly indicated that CtBP2 might be involved in EMT in HCC. Therefore, we next investigated whether the transcription factor GLI1 regulates CtBP2 expression. We stably transfected Huh7 cells with a GLI1 expressing vector or an empty control vector and observed that elevated GLI1 expression significantly enhanced CtBP2 expression (Figure 5A). Next, using MatInspector professional version 7.2, we identified several potential GLI binding sites in the CtBP2 promoter (Figure 5B). To determine whether GLI1 could directly interact with the $\mathrm{CtBP} 2$ promoter and to identify the core promoter region of $\mathrm{CtBP} 2$, we cloned four $\mathrm{CtBP} 2$ promoter fragments (pGL3-2000, -2000/-1 bp; pGL3-1307, -1307/1 bp; pGL3-675, -675/-1 bp; pGL3-1350, -1350/-652 bp) into a luciferase reporter vector and conducted a luciferase reporter assay in Huh7 cells stably transfected with the GLI1 expressing plasmid. The four reporter plasmids each displayed different levels of promoter activity, but all displayed notably stronger promoter activity than the pGL3basic control plasmid (Figure 5C). The pGL3-1307 plasmid displayed the strongest promoter activity. Remarkably, when the fragment between -1307 bp and -676 bp was deleted, the luciferase activity was almost abolished. The pGL3-1350 plasmid containing the $-1350 /-652$ bp CtBP2 promoter fragment displayed the second highest promoter activity level of the transfected Huh7 CtBP2 cells. These data suggested the existence of a critical positive regulatory element in the $-1350 /-652$ bp CtBP2 promoter fragment. To further investigate the importance of the $-1350 /-652$ bp CtBP2 promoter fragment we examined pGL3-1350 luciferase activity in Huh7 cells transfected with a GLI1 expressing plasmid (Huh7 GLI1) or a control plasmid (Huh7 Vector). We observed that elevated GLI1 expression increased the luciferase activity of the pGL3-1350 reporter plasmid (Figure 5D).

A ChIP assay further supported the hypothesis that the GLI1 protein directly interacted with and bound the $-1350 /-652$ bp CtBP2 promoter fragment. Overexpression of GLI1 increased the CtBP2 promoter occupancy of GLI1 in Huh7 cells (Figure 5E). Therefore, the results of our experiments implied that GLI1 mediates CtBP2 expression by directly binding its promoter in HCC cells.

\section{CtBP2 acted as a transcriptional co-repressor and its recruitment was critical for GLI1/SNAI1 induction of the HCC EMT phenotype}

To investigate whether CtBP2 directly induced EMT, we examined the relationship between CtBP2, SNAI1 and EMT in HCC. We abolished SNAI1 expression in Huh7 cells using SNAI1 siRNA [21] and induced CtBP2 expression with transfection of a CtBP2 expressing plasmid (Figure 6A). In the absence of SNAI1, CtBP2 overexpression did not affect the expression of E-cadherin, N-cadherin, Vimentin or Fibronectin as much as it did in cells expressing normal levels of SNAI1, as presented in Figure 6B. This result indicated that SNAI1 was required for $\mathrm{CtBP} 2$-driven $\mathrm{EMT}$ and that $\mathrm{CtBP} 2$ was not a direct mediator of EMT in HCC.

Recent studies have reported that $\mathrm{CtBP} 2$ is recruited to the E-cadherin promoter and modulates its transcription [29]. The Levine group demonstrated that the CtBP2 interaction motif P-DLS-K is present in the repression domains of SNAI1 [30]. Due to the increased SNAI1 expression we found in our previous study [21] and the enhanced CtBP2 expression observed in Huh7 GLI1 cells in this study, we conducted a Co-IP assay to observe whether CtBP2 and SNAI1 directly interact. We found that the CtBP2 protein bound the SNAI1 protein in the nucleus of Huh7 GLI1 cells (Figure 6C). To investigate the role of CtBP2 in GLI1/SNAI1 driven EMT, we used expression in Huh7 GLI1 cells (Figure 6D). We found that E-cadherin expression was increased in the siRNA CtBP2 silenced cells, that the expression of N-cadherin, Vimentin and Fibronectin were repressed, and that SNAI1 expression was not significantly altered (Figure 6E). On the other hand, CtBP2 knockdown in Huh7 Vector cells did not affect the expression of aforementioned EMT markers 


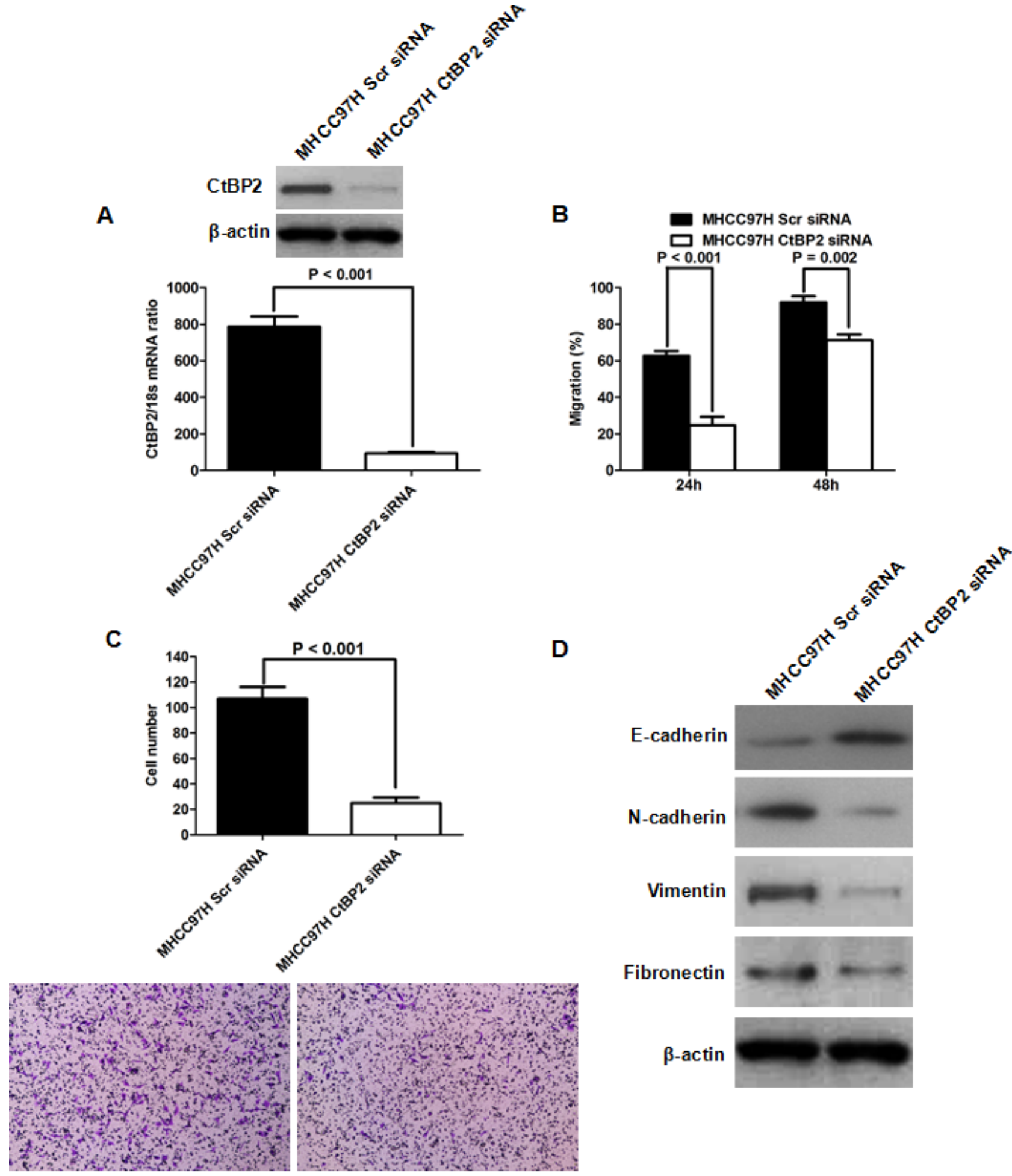

MHCC97H Scr siRNA

MHCC97H CtBP2 siRNA

Figure 4: CtBP2 knockdown inhibited MHCC97H cell migration and invasion capacities by repressing EMT. (A) Both qRT-PCR and Western blotting assays confirmed that siRNA transfection eliminated CtBP2 expression in MHCC97H cells. (B) Wound healing assays indicated that CtBP2 siRNA suppressed MHCC97H cell migration. (C) The invasive ability of MHCC97H cells was repressed by CtBP2 siRNA in Transwell invasion assays. (D) Western blotting assays demonstrated that CtBP2 suppression lead to E-cadherin upregulation and N-cadherin, Vimentin and Fibronectin downregulation in MHCC97H cells. 


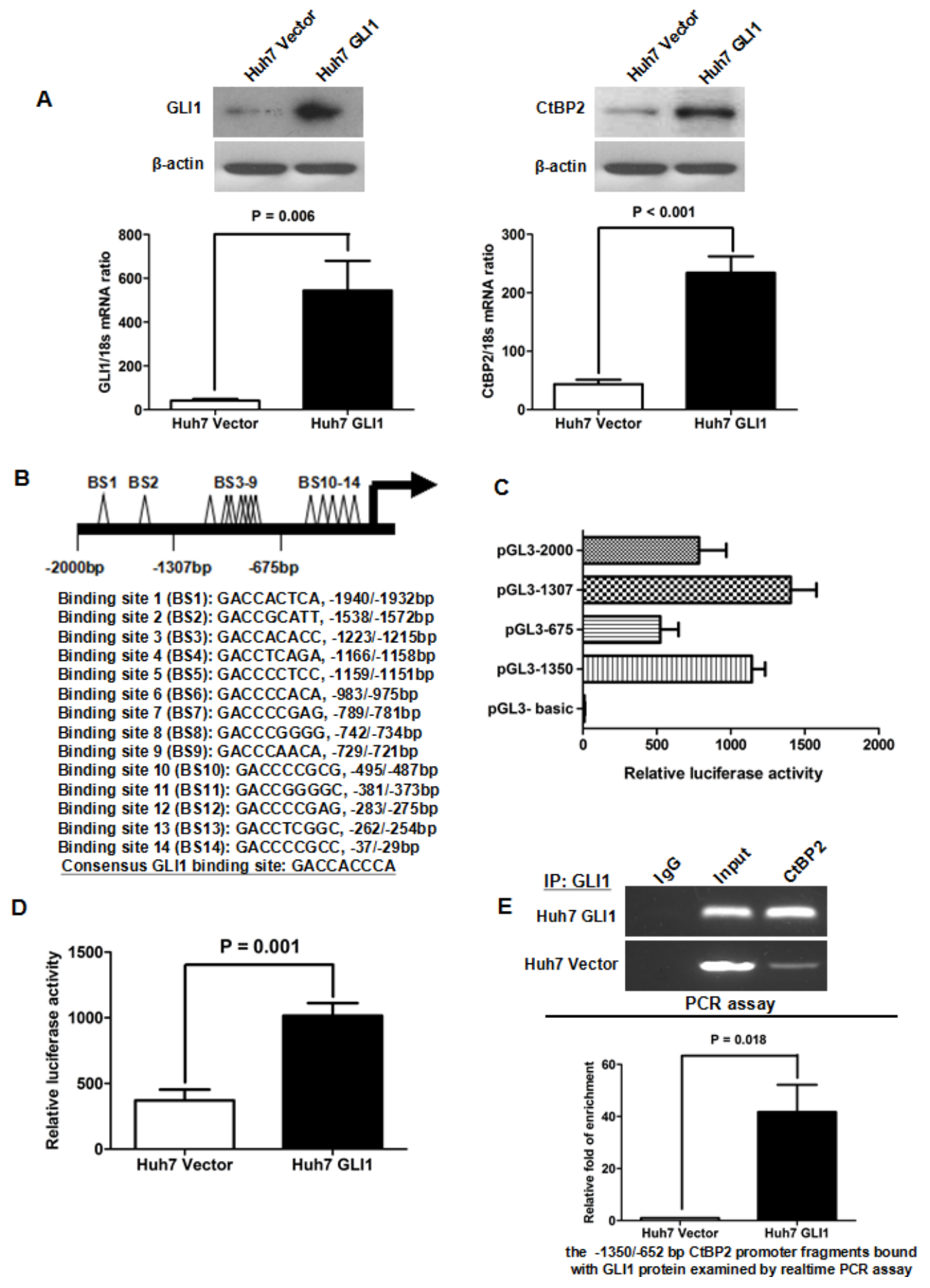

Figure 5: GLI1 increased CtBP2 expression directly by binding to its promoter. (A) Both qRT-PCR and Western blotting assays demonstrated that the expression of GLI1 (left panel) and CtBP2 (right panel) were notably elevated after GLI1 overexpression in Huh7 cells. (B) A schematic model of the locations and sequences of the 14 potential GLI1 binding sites (BS). The consensus GLI1 binding sequence is listed here. (C) Luciferase reporter assays in Huh7 GLI1 cells indicate that the pGL3-1307 (-1307/-1 bp CtBP2 promoter fragment) has the strongest promoter activity. When the fragment between $-1307 \mathrm{bp}$ and $-676 \mathrm{bp}$ was deleted, the luciferase activity greatly reduced. The pGL3-1350 (-1350/-652 bp CtBP2 promoter fragment) displayed the second highest level of promoter activity. (D) Luciferase reporter assays demonstrated that the luciferase activity of pGL3-1350 was notably enhanced after GLI1 overexpression in Huh7 cells. This supports the ability of the GLI1 protein to bind the CtBP2 promoter in the $-1350 /-652$ bp region. (E) DNA fragments capable of binding the GLI1 protein in the cell nucleus were analyzed by ChIP assay. As the upper panel shows, the PCR assay showed that the GLI1 protein was capable of directly binding to the $-1350 /-652$ bp CtBP2 promoter fragment. After equalization using RNA Polymerase II (Input), more CtBP2 promoter fragment was bound to the GLI1 protein in Huh7 GLI1 cells. Similarly, a relative rtPCR assay demonstrated that elevated GLI1 expression increased the CtBP2 promoter occupancy of GLI1 in Huh7 cells, as presented in the lower panel. 

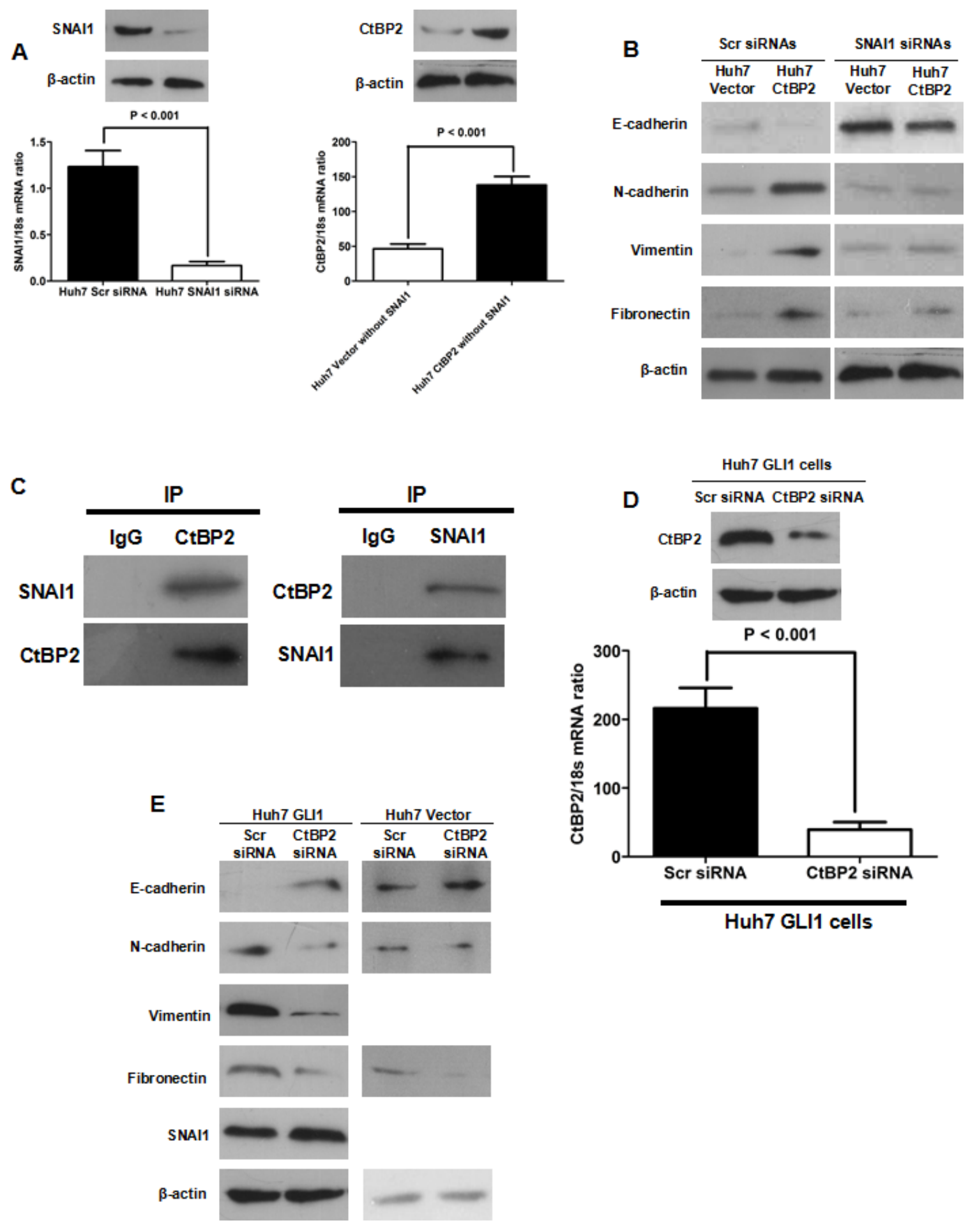

Huh7 GLI1 cells

Figure 6: CtBP2 interacted with SNAI1 and played a critical role in GLI1/SNAI1 induction of EMT. (A) Both qRTPCR and Western blotting assays demonstrated that SNAI1 siRNA abated SNAI1 expression (left panel) in Huh7 cells and that CtBP2 expression was enhanced by CtBP2 expression plasmid transfection in Huh7 cells without SNAI1. (B) Western blotting showed that CtBP2 overexpression lead to E-cadherin downregulation and N-cadherin, Vimentin and Fibronectin upregulation in Huh7 cells with normal SNAI1 expression. However, silencing SNAI1 counteracted the regulatory effect of CtBP2 on E-cadherin, N-cadherin, Vimentin and Fibronectin. (C) Co-IP assays demonstrated direct CtBP2 and SNAI1 binding in Huh7 GLI1 cells. (D) Both qRT-PCR and Western blotting verified that siRNA abolished CtBP2 expression in Huh7 GLI1 cells. (E) Western blotting assays demonstrated that CtBP2 knockdown in Huh7 GLI1 cells lead to E-cadherin upregulation and N-cadherin, Vimentin and Fibronectin downregulation, while SNAI1 expression was not significantly affected. However, silencing CtBP2 did not affect the expression of E-cadherin, N-cadherin and Fibronectin. Neither the expression of Vimentin nor the expression of SNAI1 could be detected in Huh7 Vector cells transfected with CtBP2 siRNAs or Scr siRNAs. 
clearly. These data indicated that CtBP2 bound SNAI1 as a transcriptional co-repressor and, thereby, promoted GLI1/ SNAI1 driven EMT in HCC.

\section{CtBP2 accelerated tumor growth and promoted EMT in the HCC xenograft model}

To further study the role of $\mathrm{CtBP} 2$ in $\mathrm{HCC}$ progression, we conducted HCC xenograft experiments in nude mice.

A
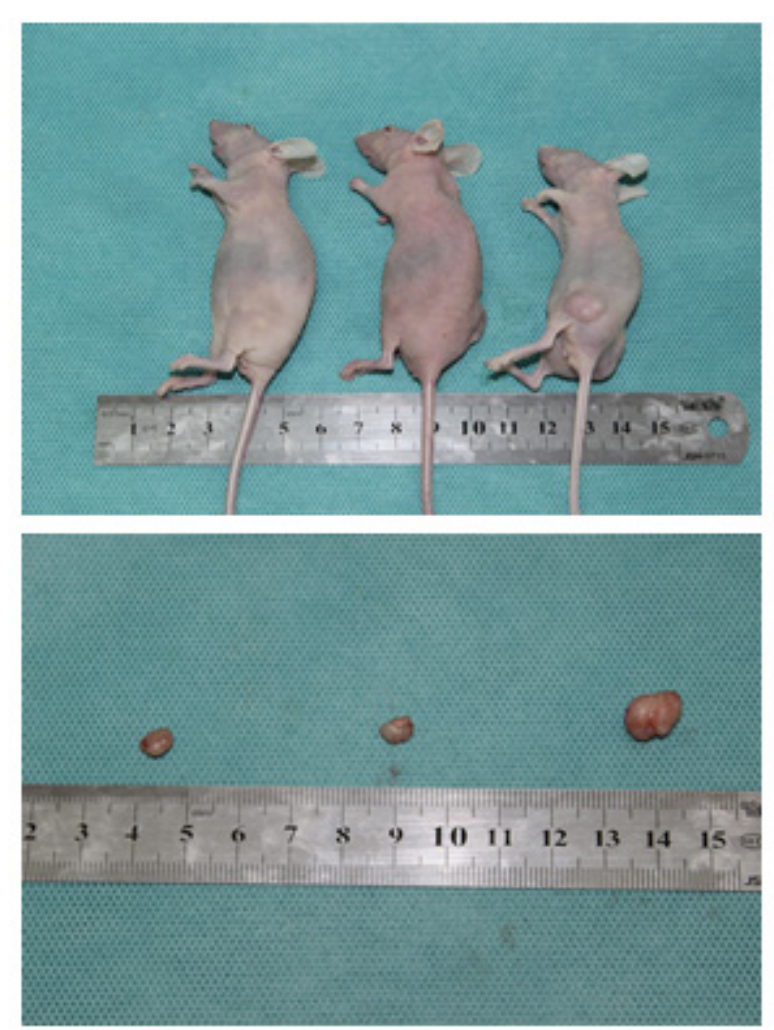

Huh7 CtBP2 and Huh7 Vector cells were subcutaneously injected into six nude mice. The growth of xenograft tumors was observed weekly using calipers and tumors were harvested after four weeks. The xenograft tumors derived from the Huh7 CtBP2 cells (Huh7 CtBP2 Group) were notably larger than those derived from the Huh7 Vector cells (Huh7 Vector Group) (Figure 7A). Unpaired Student's $t$-tests confirmed that the xenograft tumors obtained from the Huh7 CtBP2 Group were remarkably larger than the
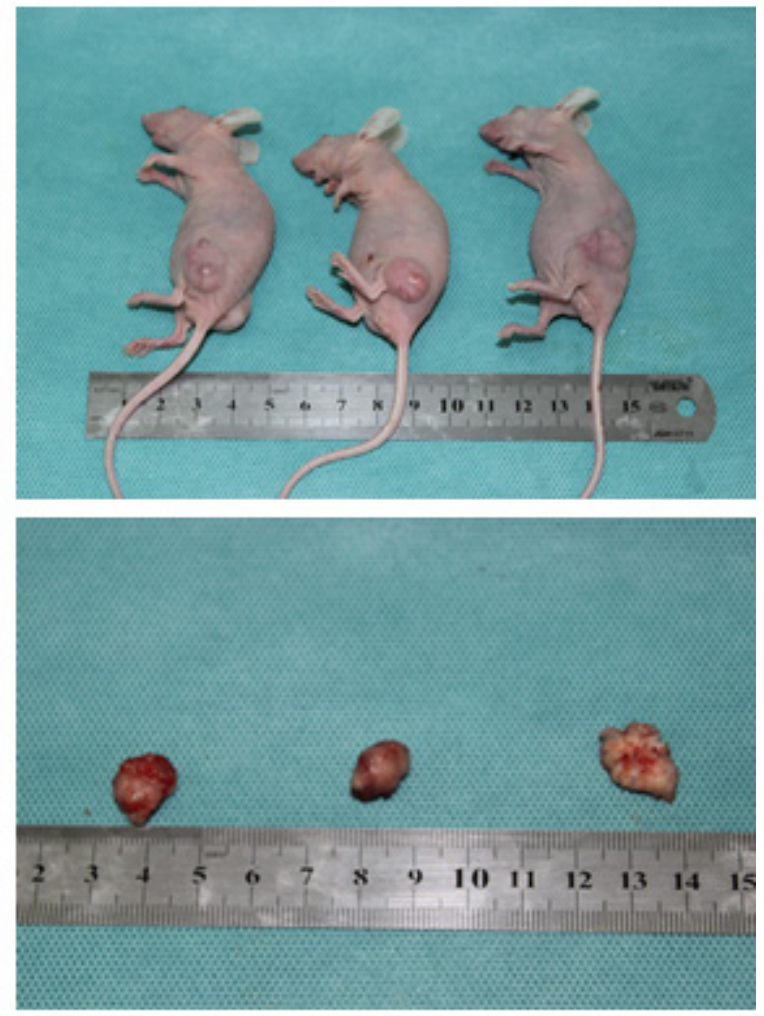

B

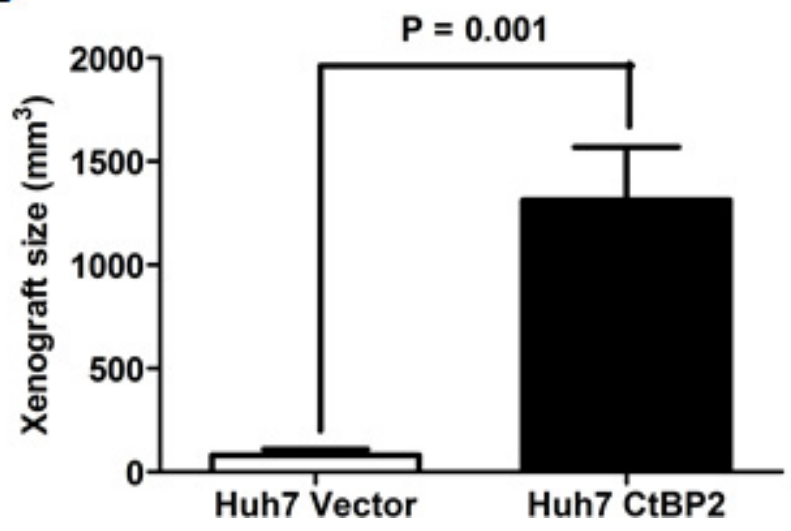

Figure 7: Elevated CtBP2 expression accelerated HCC xenograft growth and promoted EMT in HCC xenograft tissues. (A) Representative pictures of HCC xenografts from both Huh7 Vector (left panel) and Huh7 CtBP2 cells (right panel). (B) Xenograft size in the Huh7 CtBP2 group was significantly larger than in the Huh7 Vector Group $(p=0.001)$. 
tumors obtained from the Huh7 Vector Group ( $p=0.001$, Figure 7B). IHC staining demonstrated significantly stronger $\mathrm{N}$-cadherin expression and less E-cadherin expression in the Huh7 CtBP2 Group's xenograft tumors (Figure 8). We did not observe upregulation of either GLI1 or SNAI1 in the Huh7 CtBP2 Group, providing evidence that CtBP2 did not mediate either GLI1 or SNAI1 expression in xenograft HCC tumors. In summary, these results supported the hypothesis that CtBP2 promotes tumor progression and induces the EMT phenotype in HCC.

\section{DISCUSSION}

We have identified a role for $\mathrm{CtBP} 2$ in the progression of $\mathrm{HCC}$ and revealed a correlation between $\mathrm{CtBP} 2$ expression in $\mathrm{HCC}$ tissues and patient prognosis after liver resection. We report six novel discoveries in this study: (1) CtBP2 was upregulated in HCC tissues when compared to matched adjacent healthy liver tissues and CtBP2 overexpression in HCC tissues was correlated with poor patient prognosis; (2) CtBP2 overexpression promoted cell motility and invasion by inducing EMT in HCC cells; (3) GLI1 bound the CtBP2 promoter directly and increased its expression, which is potentially the underlying mechanism of CtBP2 upregulation in $\mathrm{HCC}$; (4) CtBP2 bound SNAI1, a well-known EMT inducer, and was required as a transcriptional co-repressor for GLI1/ SNAI1 driven EMT in HCC cells; (5) SNAI1 was also essential for CtBP2 induced EMT in HCC cells; (6) CtBP2 overexpression accelerated tumor growth and promoted EMT in HCC xenograft tumors. In summary, the results of this study strongly support the potential of CtBP2 as a predictive factor of patient outcome after liver resection and as a therapeutic target for the treatment of HCC.

EMT is a fundamental cellular process that is involved in epithelial tissue morphogenesis during tissue development and cancer progression. In our previous studies, we demonstrated that EMT was critical to HCC progression and regulated by the GLI1/SNAI1 axis [21, $26,27]$. To reveal the mechanism through which GLI1 and SNAI1 mediate EMT in HCC, we investigated the role of CtBP2 in GLI1/SNAI1 axis induced EMT.

CtBP2 was initially discovered as a phosphoprotein bound to the $\mathrm{C}$-terminal of the adenovirus E1A protein. It was recently reported to function as a transcriptional co-repressor that binds various transcriptional repressors with PXDLS binding motifs. In turn, these transcriptional repressors target and suppress the expression of multiple tumor repressors, including E-cadherin [31], PTEN [32] and $\mathrm{p} 16$ Ink4a [33]. Using both in vitro and in vivo experiments, we demonstrated that $\mathrm{CtBP} 2$ overexpression induced EMT in HCC and promoted cell migration and invasion. After searching the RCSB Protein Data Bank, we found that the SNAI1 protein contained the PQDLSLK motif. The presence of this motif implied that the CtBP2 protein was potentially a binding partner of the SNAI1 protein and that $\mathrm{CtBP} 2$ might function as a transcriptional co-repressor. The Co-IP results confirmed this hypothesis. Additionally, the in vitro experiments revealed that CtBP2 knockdown reversed GLI1/SNAI1 driven EMT, but CtBP2 could not induce EMT in HCC cells without SNAI1 expression. Interestingly, we found that enhanced GLI1 expression promoted CtBP2 upregulation. Fourteen potential GLI1-binding sites were observed in the CtBP2 promoter after searching the RCSB Protein Data Bank. ChIP and luciferase reporter assays suggested that GLI1 bound the $-1350 /-652$ bp CtBP2 promoter fragment. These data indicated that GLI1 directly promoted CtBP2 expression. Therefore, we concluded that SNAI1 and CtBP2 both promoted the EMT phenotype of HCC cells, could directly interact with one another and were both directly upregulated by GLI1.

This investigation also reported aberrant overexpression of CtBP2 in HCC tissues when compared to matched normal adjacent liver tissues. After analyzing the patient follow-up data, we determined that CtBP2 overexpression in $\mathrm{HCC}$ tissues predicted poor patient prognosis after liver resection. The highly significant results of this analysis suggest that $\mathrm{CtBP} 2$ could be a useful prognostic marker of patient outcome after HCC surgery. Our clinical investigation also revealed that there was positive association between GLI1 and $\mathrm{CtBP} 2$ expression in $\mathrm{HCC}$ tissues, further supporting our conclusion that GLI1 upregulates CtBP2 expression in $\mathrm{HCC}$.

In summary, as illustrated in Figure 9, we have identified a novel function for CtBP2 as a transcription co-repressor that interacts with SNAI1 and contributes to inducing the EMT phenotype of HCC cells. We found that GLI1 upregulated both SNAI1 and CtBP2. This upregulation results in the induction of EMT, and, therefore, this study characterizes the mechanism by which GLI1 modulates the EMT in HCC. Finally, CtBP2 appears to be an effective predictive factor for $\mathrm{HCC}$ outcome after liver resection and additional future studies developing $\mathrm{CtBP} 2$ as a prognostic marker, in addition to studies investigating its potential as an HCC treatment target, should be conducted.

\section{METHODS}

\section{Clinical samples}

One hundred patients diagnosed with HCC between January 2004 and June 2006 in the Department of Hepatobiliary Surgery at the First Affiliated Hospital of Xi'an Jiaotong University were recruited in this investigation. Written informed consent was obtained from all patients and all protocols in this study were approved by the Xi'an Jiaotong University Ethics Committee according to the Helsinki Declaration of 1975. None of the patients enrolled in this study had 


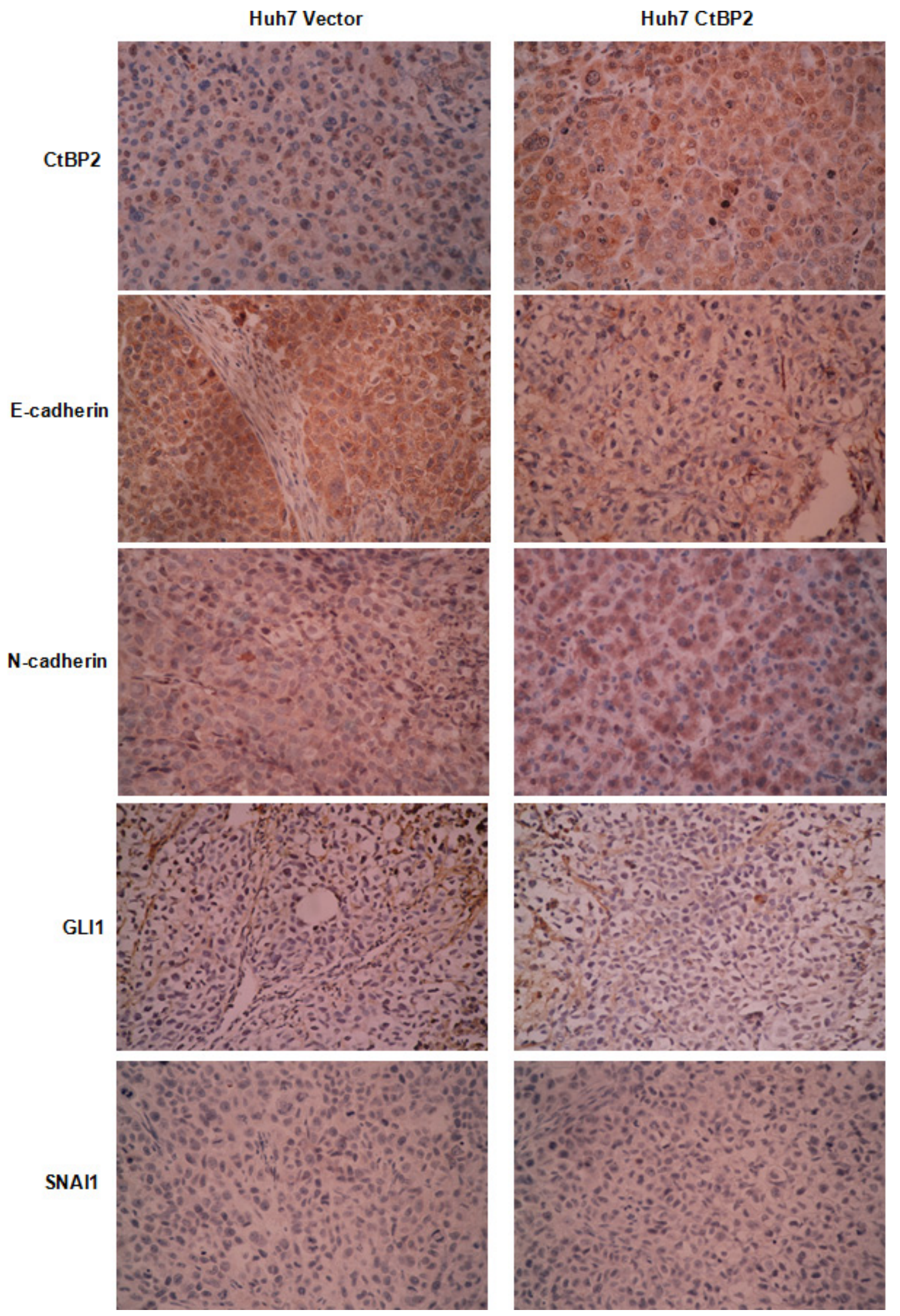

Figure 8: Compared to the Huh7 Vector group, there was more CtBP2 and N-cadherin protein expression in xenograft tissues from the Huh7 CtBP2 group, whereas E-cadherin expression was decreased. GLI1 and SNAI1 expression was undetected in xenograft tissues from either the Huh7 CtBP2 or the Huh7 Vector Group. 


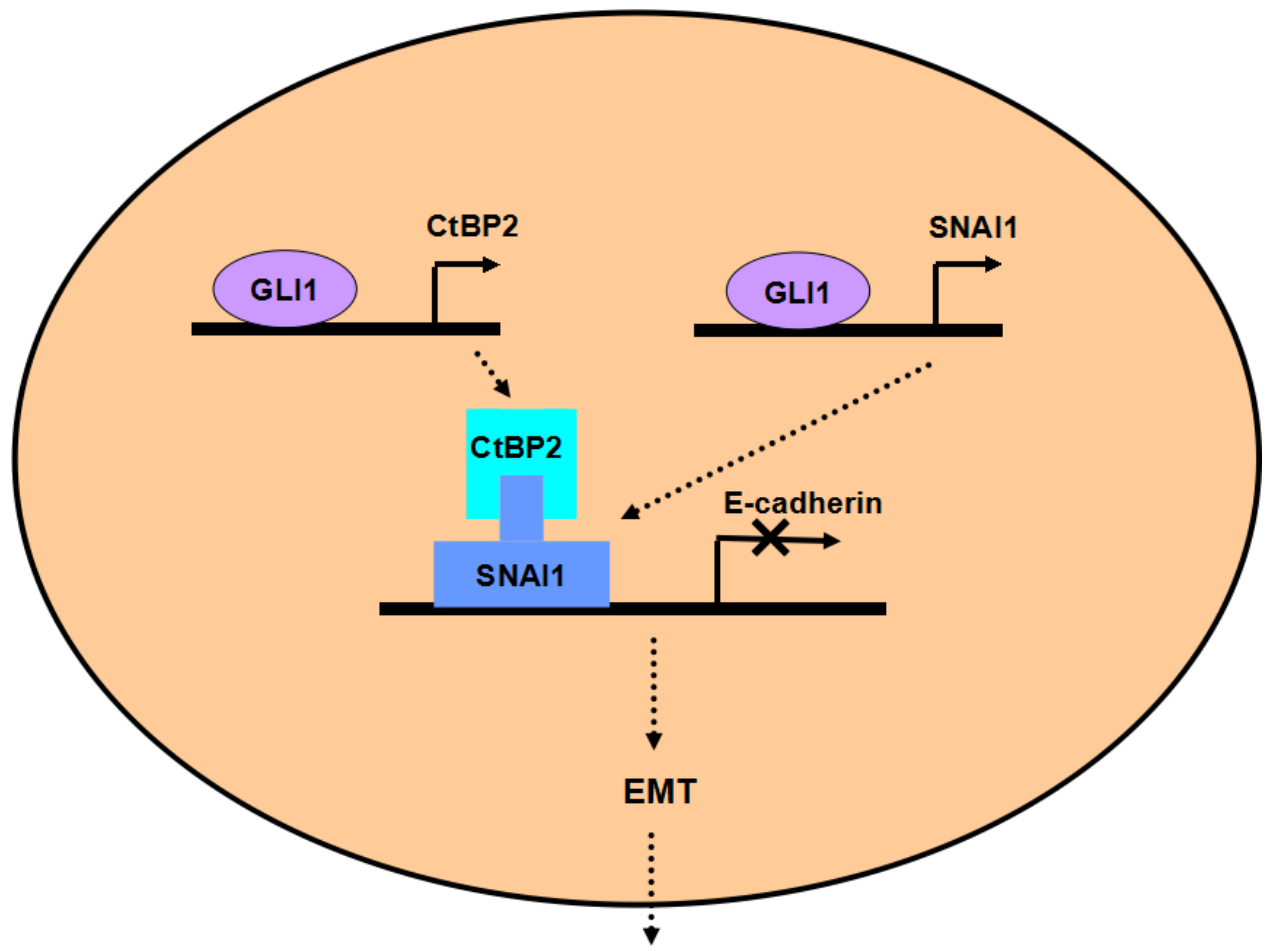

Migration and invasion of HCC cells

Figure 9: Working model of the role of CtBP2 in EMT induced by the GLI1/SNAI1 axis in HCC. GLI1 upregulates both SNAI1 and CtBP2 simultaneously. SNAI1 binds the E-cadherin promoter after binding CtBP2, leading to the EMT phenotype of HCC cells.

undergone pre-operative chemotherapy or embolization. After routine diagnostic procedures, including X-ray, abdominal ultrasonography and computed tomography, all patients received surgical treatment, including curative resection for early $\mathrm{HCC}$ and palliative resection for advanced HCC. HCC samples and paired adjacent liver samples $(>2 \mathrm{~cm}$ distance from the margin of the resected tissue) were obtained during each liver resection and immediately fixed in paraformaldehyde for immunohistochemistry. Clinical data from these $100 \mathrm{HCC}$ patients were collected from their medical records. An experienced pathologist performed the HCC diagnosis, Edmondson classification, clinical tumor-node-metastasis (TNM) grading and measured maximum tumor diameter. After surgery, all patients were followed for 15 to 120 months. Follow-up information was successfully obtained from 87 patients. The demographic features and clinicopathological data are presented in Table 1.

\section{Cell lines}

The Hep3B, PLC/PRF/5 and HepG2 human $\mathrm{HCC}$ cell lines were obtained from the Institute of Biochemistry and Cell Biology at the Chinese Academy of Sciences (Shanghai, China). The MHCC97H cells were obtained from Fudan University (Shanghai, China). The Huh7 cells were a kind gift from Prof. Kefeng Dou
(Department of Hepatobiliary Surgery, Xijing Hospital, Fourth Military Medical University). Hep3B and PLC/ PRF5 cells were cultured in complete MEM medium with $10 \%$ fetal bovine serum (FBS). MHCC97H and Huh7 cells were grown in DMEM with $10 \%$ FBS. HepG2 cells were cultured in RPMI 1640 medium with 10\% FBS. All $\mathrm{HCC}$ cells were grown in a humidified incubator with 5\% $\mathrm{CO} 2$ at $37^{\circ} \mathrm{C}$.

\section{Expression vectors}

CtBP2 expressing plasmids and GLI1 expressing plasmids were generated by cloning the respective cDNA into pCMV-Tag2B vectors purchased from Stratagene (Santa Clara, CA, USA). The sequence of both expression plasmids were confirmed by polymerase chain reaction (PCR) and Western blotting.

\section{Western blotting}

Protein expression was examined by Western blotting. Cell lysates $(50 \mu \mathrm{g})$ were prepared in RIPA buffer...The following antibodies were used: mouse monoclonal antibodies to E-cadherin (Santa Cruz, CA, USA), Vimentin (Santa Cruz), $\beta$-actin (Boster Biotechnology, Wuhan, China) and rabbit polyclonal antibodies against GLI1, SNAI1, N-cadherin and CtBP2 (Abcam, Cambridge, UK). 


\section{Quantitative real-time reverse transcription polymerase chain reaction (qRT-PCR)}

HCC cells were cultured to $80 \%$ confluence, washed with ice-cold PBS and subjected to RNA extraction using $1 \mathrm{ml}$ of Trizol (Invitrogen, NY, USA). cDNA synthesis was carried out with the High Capacity cDNA Reverse Transcription Kit from Applied Biosystems (Carlsbad, CA, USA) and ABI TaqMan Gene Expression assays were carried out for qRT-PCR using the 7300 RealTime PCR System (Applied Biosystems). 18S ribosomal RNA (rRNA) was measured as an internal control and the mRNA levels of target genes were normalized to the $18 \mathrm{~S}$ rRNA level in the same sample.

\section{Immunofluorescence and confocal microscopy}

HCC cells were plated on cover slips overnight, fixed for 20 min with $2.5 \%$ formaldehyde in PIPES buffer and then treated with $0.25 \%$ Triton X-100 for $15 \mathrm{~min}$. After incubating the slides in blocking buffer $(5 \%$ normal goat serum and $5 \%$ glycerol in D-PBS) for $1 \mathrm{~h}$ at $37^{\circ} \mathrm{C}$, they were incubated with mouse anti-E-cadherin and rabbit anti-Ncadherin antibodies at $4^{\circ} \mathrm{C}$ overnight followed by washing with PBS three times. Cover slips were then incubated with Alexa-Fluor-555 conjugated donkey anti-rabbit and AlexaFluor-488 conjugated goat anti-mouse secondary antibodies at room temperature for $60 \mathrm{~min}$, followed by DAPI nuclear counterstaining for $10 \mathrm{~min}$. Images were taken by confocal microscopy (Carl Zeiss, Oberkochen, Germany).

\section{Migration assay}

Cell mobility was determined with a wound healing assay. As described previously [27], HCC cells were grown to full confluence in 24-well plates. After the cells had grown to confluency a "wound" was created by scratching the length of the well with a $1,000 \mu$ pipette tip. Images were captured with an inverted digital camera at 0 , 24 and $48 \mathrm{~h}$. Cell migration was quantitated by measuring the width of the wound at each time point. Each assay was replicated three times.

\section{Transwell invasion assay}

Matrigel was diluted 1:3 in serum-free medium before being added to the upper chamber of a 24-well Transwell plate (Millipore, Billerica, Massachusetts, USA). HCC cells were trypsinized and counted manually under a microscope. A cell suspension of 50,000 cells $/ \mathrm{mL}$ in serum-free medium was prepared and $100 \mu \mathrm{L}$ of the suspension was loaded into the top chamber. The bottom chambers were filled with medium with $10 \%$ FBS. Invasion was halted after $24 \mathrm{hrs}$ incubation in a $37^{\circ} \mathrm{C}$ incubator $(5 \% \mathrm{CO} 2)$ by scraping the non-migrated cells off the upper chamber using a cotton swab. After being fixed in $4 \%$ paraformaldehyde, migrated cells were stained with $0.05 \%$ Crystal Violet. Migrated HCC cells were counted under an inverted light microscope.

\section{Chromatin immunoprecipitation assay (ChIP assay)}

HCC cells were cross-linked with $1 \%$ formaldehyde for $10 \mathrm{~min}$ at room temperature before the ChIP assay. The EZ-Magna ChIPTM Chromatin Immunoprecipitation Kit was purchased from Millipore for the ChIP assay. According to the manufacturer's instructions, proteins were cross-linked with $1 \%$ formaldehyde after cell lysis and DNA shearing with 15 cycles of sonication. Next, immunoprecipitation was conducted using the GLI1 antibody (Cell Signaling Technology, Danvers, MA, USA), normal rabbit IgG (Santa Cruz) as a negative control (IgG group) and anti-RNA Polymerase II antibody as the positive control (Input group). Following immunoprecipitation, cross-links were removed and immunoprecipitated DNA was purified and then amplified by qRT-PCR using the SYBR Green PCR Master Mix (Applied Biosystems). The primers used to detect the CtBP2 promoter were designed as follows: Forward: 5'-GCCGAACTCAGGGTAGTGAG-3'; Reverse: 5'-CGT CCTGACGTCTCAAGGTT-3'.

\section{Luciferase reporter assay}

Four CtBP2 promoter fragments $(-2000 /-1 \mathrm{bp}$, $-1307 /-1 \mathrm{bp},-675 /-1 \mathrm{bp}$ and $-1350 /-652 \mathrm{bp}$ from the transcription start site) were isolated by PCR amplification and ligated into the luciferase reporter vector pGL3-Basic from Promega (Fitchburg, WI, USA). Huh7 cells were plated on 24-well plates one day prior to the transfection. Huh7 cells were co-transfected with luciferase promoter reporter vectors and GLI1 expressing plasmids. After 48 hours, luciferase activity was examined using the DualLuciferase Reporter Assay System (Promega) according to the manufacturer's protocol.

\section{Co-immunoprecipitation assay (Co-IP assay)}

The co-immunoprecipitation assay was performed using Huh7 cells transfected with GLI1 expressing plasmid (Huh7 GLI1 cells). Protein lysate was extracted in immunoprecipitation buffer $(50 \mathrm{mM}$ Tris- $\mathrm{HCl}, \mathrm{pH}$ $8.0,150 \mathrm{mM} \mathrm{NaCl}, 5 \mathrm{mM}$ EDTA, 0.5\% NP-40, $2 \mu \mathrm{g} / \mathrm{ml}$ aprotinin, $1 \mu \mathrm{g} / \mathrm{ml}$ leupeptin, $1 \mathrm{mM}$ PMSF, $1 \mathrm{mM}$ sodium vanadate and $10 \mathrm{mM}$ sodium fluoride). For Co-IP, $10 \mu \mathrm{l}$ protein $\mathrm{A} / \mathrm{G}$-agarose beads pre-conjugated with the antibody against CtBP2 or SNAI1 and IgG were utilized to isolate the immunocomplexes. The antibody-conjugated beads were shaken with cell lysates at $4^{\circ} \mathrm{C}$ overnight. The next day, isolated beads were washed twice with PBS buffer and twice with RIPA buffer. The supernatant was examined by Western blotting. 


\section{Immunohistochemical (IHC) staining}

IHC staining was conducted as described previously [28]. Paraffin embeded samples were cut into $4 \mu \mathrm{m}$-thick sections, which were then deparaffinized in xylene and re-hydrated. The slices were incubated in $0.3 \%$ hydrogen peroxide for 30 min to block endogenous peroxidase activity and then antigen retrieval was performed in sodium citrate buffer for $45 \mathrm{~s}$ in a pressure cooker. After overnight blocking at $4^{\circ} \mathrm{C}$, sections were incubated with primary antibodies against CtBP2 or GLI1 at $4^{\circ} \mathrm{C}$ overnight. Bound primary antibodies were detected using biotinylated secondary antibodies (Zhongshan Goldenbridge Biotechnology Lltd. Co., Beijing, China). Next, sections were incubated with diaminobenzidine before being counterstained with hematoxylin. Finally, sections were dehydrated in alcohol and xylene.

Sections were independently evaluated by two experienced pathologists. Staining intensity was scored using four grades $(0-3)$ : 0 (negative), 1 (weakly positive), 2 (moderately positive) and 3 (strongly positive). The percentage of positive cells was also scored using five categories $(0-4)$ : a score of 0 was given for $0-5 \%, 1$ for $6-25 \%, 2$ for $26-50 \%, 3$ for $51-75 \%$, and 4 for $>75 \%$. The final staining score was obtained by multiplying the staining intensity and the percentage of positive cells.

\section{In vivo tumorigenesis assay}

$5 \times 10^{6}$ Huh7 cells were transfected using control or CtBP2 expressing plasmids before being subcutaneously injected into the flanks of 4-week-old male BALB/c nude mice (Centre of Laboratory Animals, The Medical College of Xi'an Jiaotong University, Xi'an, China). Tumor sizes were measured weekly using calipers and calculated with the following formula: volume $=\mathrm{A} \times \mathrm{B}^{2} \times 0.52$ (A, length; $\mathrm{B}$, width; all measurements were in millimeters). After four weeks, the mice were sacrificed by cervical dislocation under anesthesia. All in vivo experimental protocols were approved by the institutional animal care and use committee of the First Affiliated Hospital of Xi'an Jiaotong University.

\section{Statistical analysis}

CtBP2 protein expression levels in HCC tissues and matched normal adjacent liver tissues were compared using the Mann-Whitney $U$ test. The correlation between CtBP2 and GLI1 expression levels was analyzed using Spearman rank test. The Kaplan-Meier curves of HCC patients were assessed using the log-rank test. A $p$ value of $<0.05$ was considered significant. Multivariate analysis was performed using SPSS Version 17.0 software (SPSS Inc., Chicago, IL, USA). PRISM 5 software (GraphPad, La Jolla, CA, USA) was used for all other statistical analyses.

\section{ACKNOWLEDGMENTS}

This study was supported by grants from the National Natural Scientific Foundation of China (No. 81272645 and 81072052, to Qingguang Liu; 81301743, to Xin Zheng), the Research Fund for the doctoral Program of High Education of China from the Ministry of Education (No. 20120201120090, to Xin Zheng), the Key Science and Technology Program of Shaanxi Province (No. 2014K11-01-01-21, to Xin Zheng) and the Fundamental Research Funds for the Basic Research Operating expenses Program of Central College sponsored by Xi'an Jiaotong University, to Xin Zheng.

\section{Funding statement}

This study was supported by grants from the National Natural Scientific Foundation of China (No. 81272645 and 81072052, to Qingguang Liu; 81301743, to Xin Zheng), the Research Fund for the Doctoral Program of High Education of China from the Ministry of Education (No. 20120201120090, to Xin Zheng), the Key Science and Technology Program of Shaanxi Province (No. 2014K11-01-01-21, to Xin Zheng) and the Fundamental Research Funds for the Basic Research Operating expenses Program of Central College sponsored by Xi'an Jiaotong University, to Xin Zheng.

\section{Abbreviations}

CtBP - C-terminal binding protein, HCC hepatocellular carcinoma, GLI1 - GLI family zinc finger 1, EMT - epithelial-mesenchymal transition, PBS Phosphate buffered saline, FBS - Fetal bovine serum, Co-IP - Co-immunoprecipitation, ChIP - Chromatin Immunoprecipitation, IHC - Immunohistochemistry.

\section{Conflicts of interest}

The authors declare that they have no conflicts of interest.

\section{REFERENCES}

1. Venkatesh SK, Chandan V, Roberts LR. Liver masses: a clinical, radiologic, and pathologic perspective. Clin Gastroenterol Hepatol. 2014; 12:1414-1429.

2. Are C, Rajaram S, Are M, Raj H, Anderson BO, Chaluvarya Swamy R, Vijayakumar M, Song T, Pandey M, Edney JA, Cazap EL. A review of global cancer burden: trends, challenges, strategies, and a role for surgeons. J Surg Oncol. 2013; 107:221-226.

3. Lim KC, Chow PK, Allen JC, Siddiqui FJ, Chan ES, Tan SB. Systematic review of outcomes of liver resection for early hepatocellular carcinoma within the Milan criteria. Br J Surg. 2012; 99:1622-1629. 
4. Boyd JM, Subramanian T, Schaeper U, La Regina M, Bayley S, Chinnadurai G. A region in the C-terminus of adenovirus 2/5 E1a protein is required for association with a cellular phosphoprotein and important for the negative modulation of T24-ras mediated transformation, tumorigenesis and metastasis. EMBO J. 1993; 12:469-478.

5. Chinnadurai G. CtBP, an unconventional transcriptional corepressor in development and oncogenesis. Mol Cell. 2002; 9:213-224.

6. May T, Yang J, Shoni M, Liu S, He H, Gali R, Ng SK, Crum C, Berkowitz RS, Ng SW. BRCA1 expression is epigenetically repressed in sporadic ovarian cancer cells by overexpression of C-terminal binding protein 2. Neoplasia. 2013; 15:600-608.

7. Bergman LM, Blaydes JP. C-terminal binding proteins: emerging roles in cell survival and tumorigenesis. Apoptosis. 2006; 11:879-888.

8. Chinnadurai G. Transcriptional regulation by C-terminal binding proteins. Int J Biochem Cell Biol. 2007; 39:1593-1607.

9. Hildebrand JD, Soriano P. Overlapping and unique roles for C-terminal binding protein 1 (CtBP1) and CtBP2 during mouse development. Mol Cell Biol. 2002; 22:5296-5307.

10. Fang M, Li J, Blauwkamp T, Bhambhani C, Campbell N, Cadigan KM. C-terminal-binding protein directly activates and represses Wnt transcriptional targets in Drosophila. EMBO J. 2006; 25:2735-2745.

11. Bhambhani C, Chang JL, Akey DL, Cadigan KM. The oligomeric state of $\mathrm{CtBP}$ determines its role as a transcriptional co-activator and co-repressor of Wingless targets. EMBO J. 2011; 30:2031-2043.

12. Zhang $\mathrm{C}, \mathrm{Gao} \mathrm{C}, \mathrm{Xu} \mathrm{Y}$, Zhang Z. CtBP2 could promote prostate cancer cell proliferation through c-Myc signaling. Gene. 2014; 546:73-79.

13. Barroilhet L, Yang J, Hasselblatt K, Paranal RM, Ng SK, Rauh-Hain JA, Welch WR, Bradner JE, Berkowitz RS, $\mathrm{Ng}$ SW. C-terminal binding protein-2 regulates response of epithelial ovarian cancer cells to histone deacetylase inhibitors. Oncogene. 2013; 32:3896-3903.

14. Liu X, Yao N, Qian J, Huang H. High expression and prognostic role of CAP1 and CtBP2 in breast carcinoma: associated with E-cadherin and cell proliferation. Med Oncol. 2014; 31:878.

15. Birts CN, Harding R, Soosaipillai G, Halder T, AzimAraghi A, Darley M, Cutress RI, Bateman AC, Blaydes JP. Expression of CtBP family protein isoforms in breast cancer and their role in chemoresistance. Biol Cell. 2010; 103:1-19.

16. Atwood SX, Li M, Lee A, Tang JY, Oro AE. GLI activation by atypical protein kinase $\mathrm{C}$ iota/lambda regulates the growth of basal cell carcinomas. Nature. 2013; 494:484-488.

17. Varnat F, Siegl-Cachedenier I, Malerba M, Gervaz P, Ruiz i Altaba A. Loss of WNT-TCF addiction and enhancement of
HH-GLI1 signalling define the metastatic transition of human colon carcinomas. EMBO Mol Med. 2010; 2:440-457.

18. Gotschel F, Berg D, Gruber W, Bender C, Eberl M, Friedel M, Sonntag J, Rungeler E, Hache H, Wierling C, Nietfeld W, Lehrach H, Frischauf A, Schwartz-Albiez R, Aberger F, Korf U. Synergism between Hedgehog-GLI and EGFR signaling in Hedgehog-responsive human medulloblastoma cells induces downregulation of canonical Hedgehog-target genes and stabilized expression of GLI1. PLoS One. 2013; 8:e65403.

19. El-Zaatari M, Kao JY, Tessier A, Bai L, Hayes MM, Fontaine C, Eaton KA, Merchant JL. Gli1 deletion prevents Helicobacter-induced gastric metaplasia and expansion of myeloid cell subsets. PLoS One. 2013; 8:e58935.

20. Inaguma S, Riku M, Hashimoto M, Murakami H, Saga S, Ikeda H, Kasai K. GLI1 interferes with the DNA mismatch repair system in pancreatic cancer through BHLHE41mediated suppression of MLH1. Cancer Res. 2013; 73:7313-7323.

21. Zheng X, Vittar NB, Gai X, Fernandez-Barrena MG, Moser CD, Hu C, Almada LL, McCleary-Wheeler AL, Elsawa SF, Vrabel AM, Shire AM, Comba A, Thorgeirsson SS, Kim Y, Liu Q, Fernandez-Zapico ME, et al. The transcription factor GLI1 mediates TGFbeta1 driven EMT in hepatocellular carcinoma via a SNAI1dependent mechanism. PLoS One. 2012; 7:e49581.

22. Singh RR, Kunkalla K, Qu C, Schlette E, Neelapu SS, Samaniego F, Vega F. ABCG2 is a direct transcriptional target of hedgehog signaling and involved in stromainduced drug tolerance in diffuse large B-cell lymphoma. Oncogene. 2011; 30:4874-4886.

23. He S, Wang F, Yang L, Guo C, Wan R, Ke A, Xu L, $\mathrm{Hu}$ G, Xu X, Shen J, Wang X. Expression of DNMT1 and DNMT3a are regulated by GLI1 in human pancreatic cancer. PLoS One. 2011; 6:e27684.

24. Wang F, Xu L, Guo C, Ke A, Hu G, Xu X, Mo W, Yang L, Huang Y, He S, Wang X. Identification of RegIV as a novel GLI1 target gene in human pancreatic cancer. PLoS One. 2011; 6:e18434.

25. Agarwal NK, Qu C, Kunkalla K, Liu Y, Vega F. Transcriptional regulation of serine/threonine protein kinase (AKT) genes by glioma-associated oncogene homolog 1 . J Biol Chem. 2013; 288:15390-15401.

26. Gai X, Lu Z, Tu K, Liang Z, Zheng X. Caveolin-1 is upregulated by GLI1 and contributes to GLI1-driven EMT in hepatocellular carcinoma. PLoS One. 2014; 9:e84551.

27. Zheng X, Gai X, Wu Z, Liu Q, Yao Y. Metastasin leads to poor prognosis of hepatocellular carcinoma through partly inducing EMT. Oncol Rep. 2013; 29:1811-1818.

28. Zheng X, Yao Y, Xu Q, Tu K, Liu Q. Evaluation of gliomaassociated oncogene 1 expression and its correlation with the expression of sonic hedgehog, E-cadherin and S100a4 in human hepatocellular carcinoma. Mol Med Rep. 2010; 3:965-970. 
29. Zhang QH, Piston DW, Goodman RH. Regulation of corepressor function by nuclear NADH. Science. 2002; 295:1895-1897.

30. Nibu Y, Zhang H, Levine M. Interaction of short-range repressors with Drosophila CtBP in the embryo. Science. 1998; 280:101-104.

31. Zhao LJ, Subramanian T, Vijayalingam S, Chinnadurai G. PLDLS-dependent interaction of E1A with CtBP: regulation of CtBP nuclear localization and transcriptional functions. Oncogene. 2007; 26:7544-7551.
32. Paliwal S, Kovi RC, Nath B, Chen YW, Lewis BC, Grossman SR. The alternative reading frame tumor suppressor antagonizes hypoxia-induced cancer cell migration via interaction with the $\mathrm{COOH}$-terminal binding protein corepressor. Cancer Res. 2007; 67:9322-9329.

33. Guan C, Shi H, Wang H, Zhang J, Ni W, Chen B, Hou S, Yang X, Shen A, Ni R. CtBP2 contributes to malignant development of human esophageal squamous cell carcinoma by regulation of p16INK4A. J Cell Biochem. 2013; 114:1343-1354. 